\title{
Real-Time Object-Based Video Segmentation Using Colour Segmentation and Connected Component Labeling
}

\author{
Jau U.L. and Teh C.S. \\ Faculty of Cognitive Science and Human Development, \\ Universiti Malaysia Sarawak, \\ 94300 Kota Samarahan, Kuching, Sarawak, \\ Malaysia \\ lydia_uj@yahoo.com, csteh@fcs.unimas.my
}

\begin{abstract}
In this paper, we described two-scan connected component labeling (CCL) approach on a real-time colour video image segmentation. CCL approach is an act of region labeling and could provides opportunity to find feature of object and establish boundaries of objects which are the common properties needed by many object-based video segmentation applications. We tested the proposed technique in two experimental studies that simulates real-time object-based video segmentation. Our experiments results shown that the proposed technique could perform region labeling in a fast manner. Another advantage of the proposed technique is that it does not provide extra storage to store same label equivalence. This property gives advantage to avoid label equivalence redundancies that always happen in the CCL approach.
\end{abstract}

Keywords: Connected component labeling (CCL), real-time colour video image, region labeling, object-based video segmentation application.

\section{Introduction}

Introduced in the year of 1966 by [1], CCL is an approach that labels regions within a binary image. The component of regions is labeled with unique label which was provided by the CCL operation. The CCL approach assigned same label to the same connected component of a region and those in different connected components have different label [2]. CCL is a fundamental step in segmentation of an image objects and regions, or blobs and can be used to establish boundaries of objects, components or regions, count the number of blobs in an image [3] and to find feature of object [4]. In [5], CCL is a fundamental operation in pattern analysis (recognition) and computer (robot) vision and can be found in almost all image-based applications such as fingerprint identification, character recognition, automated inspection, target recognition, face identification, medical image analysis and computer-aided diagnosis. In this study, object-based video segmentation applications refer to the higher-level video image-based applications that require the location and/or feature 